\title{
Numerical Analysis of the Effectiveness of Two-part Piezoactuators in Vibration Reduction of Plates
}

\author{
M. WICIAK ${ }^{a, *}$ AND R. TROJANOWSKI ${ }^{b}$ \\ ${ }^{a}$ Cracow University of Technology, Institute of Mathematics, Warszawska 24, 31-155 Kraków, Poland \\ ${ }^{b}$ Department of Mechanics and Vibroacoustics, AGH University of Science and Technology \\ Al. A. Mickiewicza 30, 30-059 Krakow, Poland
}

\begin{abstract}
This paper is concerned with application of piezoelements with a step change of material properties occurring in the element's plane. Each part of an element may be controlled independently. Analytical models for different shape and location of the inner part of a two-part piezoactuator are developed. Finally, results of numerical simulations concerning effectiveness in vibration reduction of piezoactuators constructed this way are presented.
\end{abstract}

DOI: 10.12693/APhysPolA.125.A-183

PACS: 43.40.At, 43.40.Vn

\section{Introduction}

Piezoelements are important type of actuators which are used in vibration reduction. For more than 20 years [1], piezoelements have been used for reduction of noise and vibration of beams $[2-4]$ and plate structures [5-8]. The advantage of using differently shaped and distributed actuators for active control was demonstrated by many researchers. Some of the studies concern different acoustic media [5], but most of papers are devoted to homogeneous piezoactuators. Very few papers deal with different composition of piezoelements. These works use Functionally Graded Materials (FGM) [3, 910] where material properties change along the thickness of the element. This paper is concerned with piezoelements with a step change of its material properties in the element's plane.

Analytical models and results of numerical simulations on the effect of location of inner segment of twopart piezoactuators on their effectiveness in vibration reduction are presented. For this purpose, mathematical model with six special cases is discussed, and six numerical models are created, respectively. Each model is concerned with a steel plate clamped on all sides with two piezoelements attached to it. One of these elements is used for plate excitation. For each model, there is a square homogeneous piezoelement (with the base area of $1600 \mathrm{~mm}^{2}$ ) located near to the center of the plate. The second element can be a square shaped or a triangle based two-part element with its center placed near to the quarter of the length and the width of the plate (Fig. 1).

\section{Mathematical models}

It was demonstrated in [1] that vibration of a thin rectangular plate with homogeneous piezoactuator can be de-

\footnotetext{
*corresponding author; e-mail: mwiciak@pk.edu.pl
}

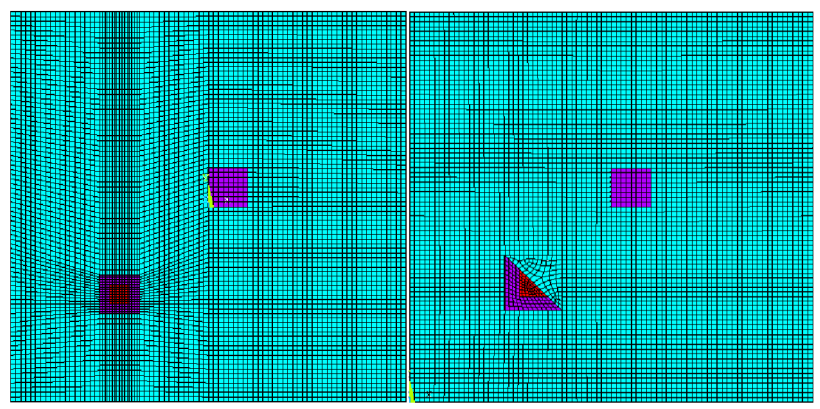

Fig. 1. Location of piezoelements.

scribed by equation

$$
D \Delta^{2} w+\mu \frac{\partial^{2}}{\partial t^{2}} w=f+F_{p e},
$$

where $w=w(t, x, y)$ is the transverse displacement, $\Delta$ is the Laplacian, $f=f(t, x, y)$ is the external excitation, $\mu=\rho h_{0}, \rho$ is the material density, $h_{0}$ is the plate thickness, $D=E h_{0}^{3} / 12\left(1-\nu^{2}\right)$ is the flexural rigidity of the plate, and $\nu$ and $E$ are the Poisson ratio and the Young's modulus, respectively. Further, $F_{p e}$ represents external forces due to homogenous actuator which, for the rectangular element shown in Fig. 2d, are given by [1]

$$
\begin{gathered}
F_{p e}=C_{0} \epsilon_{p e}\left\{\left[H_{x_{1}}-H_{x_{2}}\right] \otimes\left(\delta_{y_{1}}^{\prime}-\delta_{y_{2}}^{\prime}\right)+\left(\delta_{x_{1}}^{\prime}-\delta_{x_{2}}^{\prime}\right)\right. \\
\left.\otimes\left[H_{y_{1}}-H_{y_{2}}\right]\right\},
\end{gathered}
$$

and according to [8], for triangular actuator (Fig. 3d) by

$$
\begin{gathered}
F_{p e}=C_{0} \epsilon_{p e}\left\{\left(\delta_{x_{1}}^{\prime}-\delta_{x_{2}}^{\prime}\right) \otimes\left[H_{y_{1}}-H_{f_{(\cdot)}}\right]-2\left(\delta_{x_{1}}-\delta_{x_{2}}\right)\right. \\
\left.\otimes \delta_{f(\cdot)}+\left[H_{x_{1}}-H_{x_{2}}\right] \otimes\left(\delta_{y_{1}}^{\prime}-2 \delta_{f(\cdot)}^{\prime}\right)\right\},
\end{gathered}
$$

where $C_{0}=E I K^{f}$ denotes the piezoelectric strain-plate moment coupling term, $K^{f}$ is the material-geometric constant, $\epsilon_{p e}=\left(d_{31} V\right) / h$ is the magnitude of the induced strain, $d_{31}$ is the piezoelectric-strain constant, $V$ 
is the applied voltage, $f(x)=-x+x_{1}+y_{2}$. Since in formulas for $F_{p e}$ in Eqs. (2) and (3) there are distributions: $\left[H_{x_{i}}\right](\varphi)=\int_{x_{i}}^{+\infty} \varphi(x) \mathrm{d} x, \delta_{x_{i}}(\varphi)=\varphi\left(x_{i}\right)$ for any test function $\varphi \in D(R)$, and their derivatives (in the distributional sense), symbol $\otimes$ denotes the tensor product of distributions in Eq. (2) and the generalized tensor product of distribution and the distribution value function in Eq. (3), [8]. Consequently, the solution of Eq. (1) is also understood in the distributional sense [8].
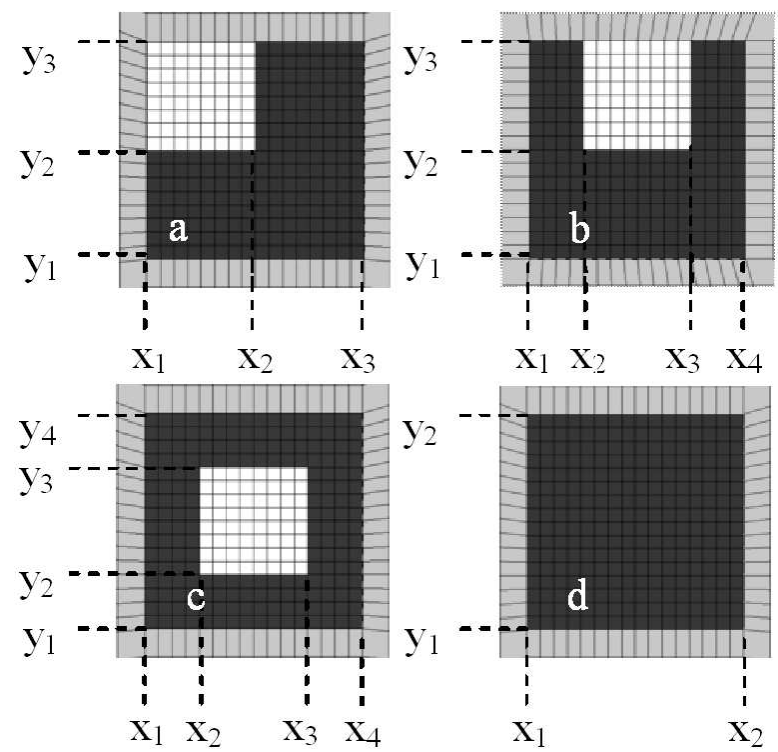

Fig. 2. The modeled piezoactuators. From left to right: inner segment at the corner of the element, inner segment at the side of the element, inner segment in the center of the element.

Consider now a two-part square shaped element with a step change in composition and different position of the inner segment. Three different positions of such inner segment were taken into consideration, as shown in Fig. 2. External forces due to two-part piezoelectric can be expressed as

$$
F_{p e}=\frac{\partial^{2} m_{x}}{\partial x^{2}}+\frac{\partial^{2} m_{y}}{\partial y^{2}}
$$

while

$$
m_{x}=m_{y}=m=\sum_{i=1}^{2} C^{i} \chi_{i},
$$

are internal moments across the piezoelectric element, where $C^{i}=C_{0}^{i} \epsilon_{p e}^{i}$, and the $i$-th index is used to denote appropriate values for the $i$-th sub-region, $i=1$ for the outer part, and $i=2$ for the inner one, while $\chi_{i}$ is a characteristic function defined on the area of the piezoelectric actuator $\Gamma_{i}$,

$$
\chi_{i}(x, y)=\left\{\begin{array}{l}
1 \text { if }(x, y) \in \Gamma_{i} \\
0 \text { if }(x, y) \notin \Gamma_{i}
\end{array}\right.
$$

for $i=1,2$, where $\Gamma_{1}=\left\{(x, y): x_{1} \leq x \leq x_{3}, y_{1} \leq y \leq\right.$ $y_{2}$ or $\left.x_{2} \leq x \leq x_{3}, y_{2} \leq y \leq y_{3}\right\}, \Gamma_{2}=\left\{(x, y): x_{1} \leq x \leq\right.$ $\left.x_{2}, y_{2} \leq y \leq y_{3}\right\}$ for the first position of the inner part (Fig. 2a), while $\Gamma_{2}=\left\{(x, y): x_{2} \leq x \leq x_{3}, y_{2} \leq y \leq y_{3}\right\}$, $\Gamma_{1}=\left\{(x, y): x_{1} \leq x \leq x_{4}, y_{1} \leq y \leq y_{2}\right.$ or $\left(x_{1} \leq x \leq\right.$ $x_{2}$ or $\left.\left.x_{3} \leq x \leq x_{4}\right), y_{2} \leq y \leq y_{3}\right\}$ for the second one (Fig. 2b), and $\Gamma_{1}=\left\{(x, y): x_{1} \leq x \leq x_{4},\left(y_{1} \leq y \leq\right.\right.$ $y_{2}$ or $\left.y_{3} \leq y \leq y_{4}\right)$ or $\left(x_{1} \leq x \leq x_{2}\right.$ or $\left.x_{3} \leq x \leq x_{4}\right), y_{2} \leq$ $\left.y \leq y_{3}\right\}$ for the third case (Fig. 2c).

In the first case, when the inner segment is placed in a corner of the outer one (Fig. 2a), the internal moments across the piezoelement are given by

$$
\begin{aligned}
m & =C^{1}\left\{\left[H_{x_{1}}-H_{x_{3}}\right] \otimes\left[H_{y_{1}}-H_{y_{2}}\right]+\left[H_{x_{2}}-H_{x_{3}}\right]\right. \\
& \left.\otimes\left[H_{y_{2}}-H_{y_{3}}\right]\right\}+C^{2}\left\{\left[H_{x_{1}}-H_{x_{2}}\right] \otimes\left[H_{y_{2}}-H_{y_{3}}\right]\right\},
\end{aligned}
$$

and consequently

$$
\begin{aligned}
& F_{p e}=C^{1}\left\{\left(\delta_{x_{1}}^{\prime}-\delta_{x_{3}}^{\prime}\right) \otimes\left[H_{y_{1}}-H_{y_{2}}\right]+\left(\delta_{x_{2}}^{\prime}-\delta_{x_{3}}^{\prime}\right)\right. \\
& \otimes\left[H_{y_{2}}-H_{y_{3}}\right]+\left[H_{x_{1}}-H_{x_{3}}\right] \\
& \left.\otimes\left(\delta_{y_{1}}^{\prime}-\delta_{y_{2}}^{\prime}\right)+\left[H_{x_{2}}-H_{x_{3}}\right] \otimes\left(\delta_{y_{2}}^{\prime}-\delta_{y_{3}}^{\prime}\right)\right\} \\
& +C^{2}\left\{\left(\delta_{x_{1}}^{\prime}-\delta_{x_{2}}^{\prime}\right) \otimes\left[H_{y_{2}}-H_{y_{3}}\right]+\left[H_{x_{1}}-H_{x_{2}}\right]\right. \\
& \left.\otimes\left(\delta_{y_{2}}^{\prime}-\delta_{y_{3}}^{\prime}\right)\right\} .
\end{aligned}
$$

In the second case, when the inner segment is placed on one side of the outer one (Fig. 2b), the internal moments across the piezoelement can be expressed by

$$
\begin{aligned}
m & =C^{1}\left\{\left[H_{x_{1}}-H_{x_{4}}\right] \otimes\left[H_{y_{1}}-H_{y_{2}}\right]\right. \\
& \left.+\sum_{k=1}^{4}(-1)^{k+1}\left[H_{x_{k}}\right] \otimes\left[H_{y_{2}}-H_{y_{3}}\right]\right\} \\
& +C^{2}\left\{\left[H_{x_{2}}-H_{x_{3}}\right] \otimes\left[H_{y_{2}}-H_{y_{3}}\right]\right\} .
\end{aligned}
$$

Thus

$$
\begin{aligned}
& F_{p e}=C^{1}\left\{\left(\delta_{x_{1}}^{\prime}-\delta_{x_{4}}^{\prime}\right) \otimes\left[H_{y_{1}}-H_{y_{2}}\right]+\left[H_{x_{1}}-H_{x_{4}}\right]\right. \\
& \quad \otimes\left(\delta_{y_{1}}^{\prime}-\delta_{y_{2}}^{\prime}\right)+\sum_{k=1}^{4}(-1)^{k+1} \delta_{x_{k}}^{\prime} \otimes\left[H_{y_{2}}-H_{y_{3}}\right] \\
& \left.\quad+\sum_{k=1}^{4}(-1)^{k+1}\left[H_{x_{k}}\right] \otimes\left(\delta_{y_{2}}^{\prime}-\delta_{y_{3}}^{\prime}\right)\right\} \\
& \quad+C^{2}\left\{\left(\delta_{x_{2}}^{\prime}-\delta_{x_{3}}^{\prime}\right) \otimes\left[H_{y_{2}}-H_{y_{3}}\right]+\left[H_{x_{2}}-H_{x_{3}}\right]\right. \\
& \left.\otimes\left(\delta_{y_{2}}^{\prime}-\delta_{y_{3}}^{\prime}\right)\right\} .
\end{aligned}
$$

Finally, in the case of central position of the inner part of a piezoelement, as shown in Fig. 2c, one can obtain

$$
\begin{aligned}
m & =C^{1}\left\{\sum_{k=1}^{4}(-1)^{k+1}\left[H_{x_{1}}-H_{x_{4}}\right] \otimes\left[H_{y_{k}}\right]\right. \\
& \left.+\sum_{k=1}^{4}(-1)^{k+1}\left[H_{x_{k}}\right] \otimes\left[H_{y_{2}}-H_{y_{3}}\right]\right\} \\
& \times+C^{2}\left\{\left[H_{x_{2}}-H_{x_{3}}\right] \otimes\left[H_{y_{2}}-H_{y_{3}}\right]\right\},
\end{aligned}
$$

and 


$$
\begin{aligned}
F_{p e} & =C^{1}\left\{\sum_{k=1}^{4}(-1)^{k+1}\left(\delta_{x_{1}}^{\prime}-\delta_{x_{4}}^{\prime}\right) \otimes\left[H_{y_{k}}\right]\right. \\
& +\sum_{k=1}^{4}(-1)^{k+1}\left[H_{x_{1}}-H_{x_{4}}\right] \otimes \delta_{y_{k}}^{\prime}+\sum_{k=1}^{4}(-1)^{k+1} \delta_{x_{k}}^{\prime} \\
& \left.\otimes\left[H_{y_{2}}-H_{y_{3}}\right]+\sum_{k=1}^{4}(-1)^{k+1}\left[H_{x_{k}}\right] \otimes\left(\delta_{y_{2}}^{\prime}-\delta_{y_{3}}^{\prime}\right)\right\} \\
& +C^{2}\left\{\left(\delta_{x_{2}}^{\prime}-\delta_{x_{3}}^{\prime}\right) \otimes\left[H_{y_{2}}-H_{y_{3}}\right]+\left[H_{x_{2}}-H_{x_{3}}\right]\right. \\
& \left.\otimes\left(\delta_{y_{2}}^{\prime}-\delta_{y_{3}}^{\prime}\right)\right\} .
\end{aligned}
$$
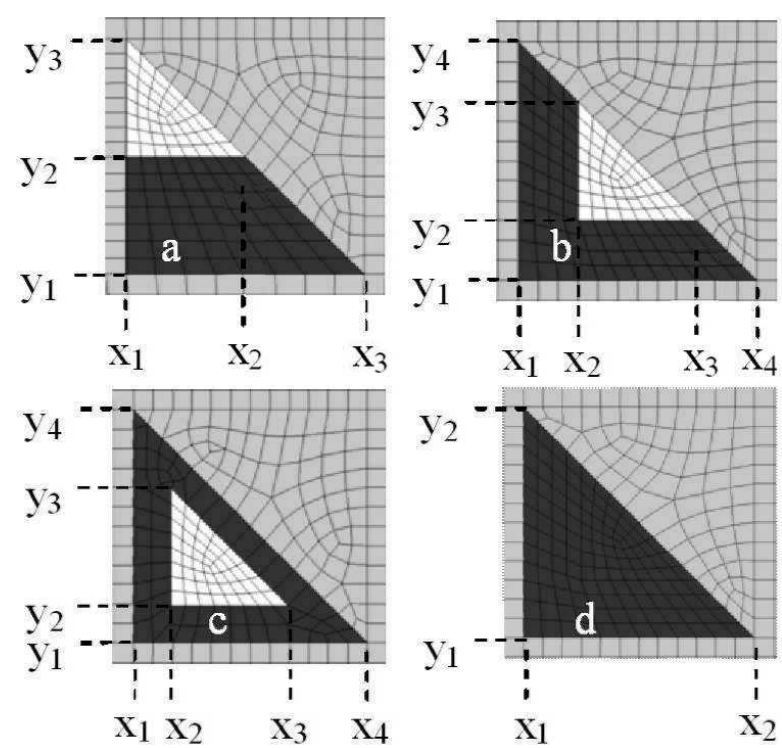

Fig. 3. The modeled piezoactuators. From left to right: inner segment at the corner of the element, inner segment at the side of the element, inner segment in the center of the element.

Now let us turn to the case of two-part piezoelement of triangle shape. It simplifies the computation and causes no loss of generality to assume that the piezoelement is a right isosceles triangle. Three different positions of the inner segment, as shown in Fig. 3, will be discussed. For the first case (Fig. 3a) sets $\Gamma_{1}, \Gamma_{2}$ in Eq. (6) are defined as $\Gamma_{1}=\left\{(x, y): x_{1} \leq x \leq\right.$ $x_{2}, y_{1} \leq y \leq y_{2}$ or $\left.x_{2} \leq x \leq x_{3}, y_{1} \leq y \leq f(x)\right\}$ $\Gamma_{2}=\left\{(x, y): x_{1} \leq x \leq x_{2}, y_{2} \leq y \leq f(x)\right\}$, where $f(x)=-x+b$, and $b=x_{1}+y_{3}$. Consequently,

$$
\begin{aligned}
m & =C^{1}\left\{\left[H_{x_{1}}-H_{x_{2}}\right] \otimes\left[H_{y_{1}}-H_{y_{2}}\right]+\left[H_{x_{2}}-H_{x_{3}}\right]\right. \\
& \left.\otimes\left[H_{y_{1}}-H_{f(\cdot)}\right]\right\}+C^{2}\left\{\left[H_{x_{1}}-H_{x_{2}}\right]\right. \\
& \left.\otimes\left[H_{y_{2}}-H_{f(\cdot)}\right]\right\}
\end{aligned}
$$

where the tensor product in the second and the third term is understood in the generalized sense as a tensor product of a distribution and a distribution-valued function [8], namely if $S \in D^{\prime}(R)$ and $T: R \ni x \mapsto T(x) \in$ $D^{\prime}(R)$, then the generalized tensor product $S \otimes T$ is a distribution that belongs to $D^{\prime}\left(R^{2}\right)$ and is defined as
$(S \otimes T)(\varphi)=S(x \mapsto T(\varphi(x, \cdot)))$

for any $\varphi \in D\left(R^{2}\right)$. Bearing in mind formulas for derivatives of generalized tensor product $S \otimes T$, [8],

$$
\begin{aligned}
\frac{\partial^{k}}{\partial x^{k}}(S \otimes T) & =\sum_{i=0}^{k}\left(\begin{array}{c}
k \\
i
\end{array}\right) \frac{\mathrm{d}^{i}}{\mathrm{~d} x^{i}} S \otimes T^{(k-i)}(\cdot), \\
\frac{\partial^{k}}{\partial y^{k}}(S \otimes T) & =S \otimes \frac{\mathrm{d}^{k}}{\mathrm{~d} y^{k}} T(\cdot),
\end{aligned}
$$

for any $k \in N$, where symbols $\partial^{k} / \partial x^{k}, \mathrm{~d}^{i} / \mathrm{d} x^{i}, \partial^{k} / \partial y^{k}$, $\mathrm{d}^{k} / \mathrm{d} y^{k}$, denote derivatives of distributions, while $T^{(j)}$ means the $j$-th derivative of the map $T$, i.e.

$$
T^{(j)}(x)(\varphi)=\frac{\mathrm{d}^{j}}{\mathrm{~d} x^{j}}(T(x) \varphi)
$$

for any $\varphi \in D(R)$, one can obtain

$$
\begin{aligned}
& F_{p e}=C^{1}\left\{\left(\delta_{x_{1}}^{\prime}-\delta_{x_{2}}^{\prime}\right) \otimes\left[H_{y_{1}}-H_{y_{2}}\right]+\left(\delta_{x_{2}}^{\prime}-\delta_{x_{3}}^{\prime}\right)\right. \\
& \otimes\left[H_{y_{1}}-H_{f(\cdot)}\right]+\left[H_{x_{1}}-H_{x_{2}}\right] \otimes\left(\delta_{y_{1}}^{\prime}-\delta_{y_{2}}^{\prime}\right) \\
& \left.\quad+\left[H_{x_{2}}-H_{x_{3}}\right] \otimes\left(\delta_{y_{1}}^{\prime}-2 \delta_{f(\cdot)}^{\prime}\right)-2\left(\delta_{x_{2}}-\delta_{x_{3}}\right) \otimes \delta_{f(\cdot)}\right\} \\
& +C^{2}\left\{\left(\delta_{x_{1}}^{\prime}-\delta_{x_{2}}^{\prime}\right) \otimes\left[H_{y_{2}}-H_{f(\cdot)}\right]-2\left(\delta_{x_{1}}-\delta_{x_{2}}\right)\right. \\
& \left.\otimes \delta_{f(\cdot)}+\left[H_{x_{1}}-H_{x_{2}}\right] \otimes\left(\delta_{y_{2}}^{\prime}-2 \delta_{f(\cdot)}^{\prime}\right)\right\} .
\end{aligned}
$$

In the case when an inner segment is located as in Fig. 3b, $\Gamma_{1}=\left\{(x, y):\left(x_{1} \leq x \leq x_{2}\right.\right.$ or $x_{3} \leq x \leq$ $\left.x_{4}\right), y_{1} \leq y \leq f(x)$ or $\left.x_{2} \leq x \leq x_{3}, y_{1} \leq y \leq y_{2}\right\}$ and $\Gamma_{2}=\left\{(x, y): x_{2} \leq x \leq x_{3}, y_{2} \leq y \leq f(x)\right\}$, where similarly to the first case $f(x)=-x+b, b=x_{1}+y_{4}$. Thus

$$
\begin{aligned}
m & =C^{1}\left\{\sum_{k=1}^{4}(-1)^{k+1}\left[H_{x_{k}}\right] \otimes\left[H_{y_{1}}-H_{f(\cdot)}\right]\right. \\
& \left.+\left[H_{x_{2}}-H_{x_{3}}\right] \otimes\left[H_{y_{1}}-H_{y_{2}}\right]\right\} \\
& +C^{2}\left\{\left[H_{x_{1}}-H_{x_{3}}\right] \otimes\left[H_{y_{2}}-H_{f(\cdot)}\right]\right\} .
\end{aligned}
$$

Consequently, on account of Eqs. (15), (16), and (3),

$$
\begin{aligned}
& F_{p e}=C^{1}\left\{\sum_{k=1}^{4}(-1)^{k+1} \delta_{x_{k}}^{\prime} \otimes\left[H_{y_{1}}-H_{f(\cdot)}\right]+\left(\delta_{x_{2}}^{\prime}\right.\right. \\
& \left.-\delta_{x_{3}}^{\prime}\right) \otimes\left[H_{y_{1}}-H_{y_{2}}\right]-2 \sum_{k=1}^{4}(-1)^{k+1} \delta_{x_{k}} \otimes \delta_{f(\cdot)} \\
& +\sum_{k=1}^{4}(-1)^{k+1}\left[H_{x_{k}}\right] \otimes\left(\delta_{y_{1}}^{\prime}-2 \delta_{f(\cdot)}^{\prime}\right)+\left[H_{x_{2}}-H_{x_{3}}\right] \\
& \left.\otimes\left(\delta_{y_{1}}^{\prime}-\delta_{y_{2}}^{\prime}\right)\right\}+C^{2}\left\{\left(\delta_{x_{1}}^{\prime}-\delta_{x_{3}}^{\prime}\right) \otimes\left[H_{y_{2}}-H_{f(\cdot)}\right]\right. \\
& -2\left(\delta_{x_{1}}-\delta_{x_{3}}\right) \otimes \delta_{f(\cdot)}+\left[H_{x_{1}}-H_{x_{3}}\right] \\
& \left.\otimes\left(\delta_{y_{2}}^{\prime}-2 \delta_{f(\cdot)}^{\prime}\right)\right\} .
\end{aligned}
$$

Finally, consider the case of central position of the inner segment, as shown in Fig. 3c. Sets $\Gamma_{1}, \Gamma_{2}$ in (6) are now given in the form $\Gamma_{1}=\left\{(x, y): x_{1} \leq x \leq x_{2}, y_{1} \leq\right.$ $y \leq f_{1}(x)$ or $x_{2} \leq x \leq x_{3},\left(y_{1} \leq y \leq y_{2}\right.$ or $f_{2}(x) \leq$ $\left.y \leq f_{1}(x)\right)$ or $\left.x_{3} \leq x \leq x_{4}, y_{1} \leq y \leq f_{1}(x)\right\}$ and $\Gamma_{2}=\left\{(x, y): x_{2} \leq x \leq x_{3}, y_{2} \leq y \leq f_{2}(x)\right\}$, where $f_{i}(x)=-x+b_{i}$ for $i=1,2, b_{1}=x_{1}+y_{4}, b_{2}=x_{2}+y_{3}$, 
and in consequence,

$$
\begin{aligned}
m & =C^{1}\left(\sum_{k=1}^{4}(-1)^{k+1}\left[H_{x_{k}}\right] \otimes\left[H_{y_{1}}-H_{f_{1}(\cdot)}\right]\right. \\
& \left.+\left[H_{x_{2}}-H_{x_{3}}\right] \otimes\left\{\left[H_{y_{1}}-H_{y_{2}}\right]+\left[H_{f_{2}(\cdot)}-H_{f_{1}(\cdot)}\right]\right\}\right) \\
& +C^{2}\left\{\left[H_{x_{2}}-H_{x_{3}}\right] \otimes\left[H_{y_{2}}-H_{f_{2}(\cdot)}\right]\right\} .
\end{aligned}
$$

Using now formulae for derivatives of the generalized tensor product in Eqs. (15) and (16) and reducing similar terms, one can obtain

$$
\begin{aligned}
& F_{p e}=C^{1}\left\{\left(\delta_{x_{1}}^{\prime}-\delta_{x_{4}}^{\prime}\right) \otimes\left[H_{y_{1}}-H_{f_{1}(\cdot)}\right]+\left(\delta_{x_{2}}^{\prime}-\delta_{x_{3}}^{\prime}\right)\right. \\
& \otimes\left[H_{f_{2}(\cdot)}-H_{y_{2}}\right]-2\left(\delta_{x_{1}}-\delta_{x_{4}}\right) \otimes \delta_{f_{1}(\cdot)} \\
& +2\left(\delta_{x_{2}}-\delta_{x_{3}}\right) \otimes \delta_{f_{2}(\cdot)}+\left[H_{x_{1}}-H_{x_{4}}\right] \otimes\left(\delta_{y_{1}}^{\prime}\right. \\
& \left.\left.-2 \delta_{f_{1}(\cdot)}^{\prime}\right)+\left[H_{x_{2}}-H_{x_{3}}\right] \otimes\left(2 \delta_{f_{2}(\cdot)}^{\prime}-\delta_{y_{2}}^{\prime}\right)\right\} \\
& +C^{2}\left\{\left(\delta_{x_{2}}^{\prime}-\delta_{x_{3}}^{\prime}\right) \otimes\left[H_{y_{2}}-H_{f_{2}(\cdot)}\right]-2\left(\delta_{x_{2}}-\delta_{x_{3}}\right)\right. \\
& \left.\otimes \delta_{f_{2}(\cdot)}+\left[H_{x_{2}}-H_{x_{3}}\right] \otimes\left(\delta_{y_{2}}^{\prime}-2 \delta_{f_{2}(\cdot)}^{\prime}\right)\right\} .
\end{aligned}
$$

\section{Numerical simulations}

In this section, results of numerical simulations concerning six different cases are presented. Each actuator had a base area of $1600 \mathrm{~mm}^{2}$ with the inner segment having the base area of $400 \mathrm{~mm}^{2}$. There were three models developed for square based actuators and three for right isosceles triangle based actuators. In each model location of the inner segment was different. Fig. 2 and Fig. 3 show the modeled piezoactuators. Elements used for modeling the plate and the piezo elements and material properties were as follows:

- Plate: SOLSH190 - $E=1.93 \times 10^{11} \mathrm{~Pa}$, $\nu=0.29, \rho=7800 \mathrm{~kg} / \mathrm{m}^{3}$;

- Piezo element plates used for excitation: SOLID226 with PZ 28;

- Piezo element plates used for actuators: SOLID226 with PZ 28 and PZ 29;

- Properties of PZ 28: $\rho=7.70 \times 10^{3} \mathrm{~kg} / \mathrm{m}^{3}$, $d_{31}=-1.14 \times 10^{-10} \mathrm{~m} / \mathrm{V}, d_{33}=2.75 \times 10^{-10} \mathrm{~m} / \mathrm{V}$ $d_{15}=4.03 \times 10^{-10} \mathrm{~m} / \mathrm{V}, s_{11}^{\mathrm{E}}=1.26 \times 10^{-11} \mathrm{~Pa}^{-1}$, $s_{33}^{\mathrm{E}}=1.83 \times 10^{-11} \mathrm{~Pa}^{-1}, s_{12}^{\mathrm{E}}=-3.71 \times 10^{-12} \mathrm{~Pa}^{-1}$, $s_{13}^{\mathrm{E}}=6.60 \times 10^{-12} \mathrm{~Pa}^{-1}, s_{44}^{\mathrm{E}}=3.77 \times 10^{-11} \mathrm{~Pa}^{-1}$, $s_{66}^{\mathrm{E}}=3.26 \times 10^{-11} \mathrm{~Pa}^{-1}, \frac{\varepsilon_{11}^{S}}{\varepsilon_{0}}=1.22 \times 10^{3}$, $\frac{\varepsilon_{33}^{S}}{\varepsilon_{0}}=9.90 \times 10^{2}$;

- Properties of PZ 29: $\rho=7.46 \times 10^{3} \mathrm{~kg} / \mathrm{m}^{3}$, $d_{31}=-2.43 \times 10^{-10} \mathrm{~m} / \mathrm{V}, d_{33}=5.74 \times 10^{-10} \mathrm{~m} / \mathrm{V}$, $d_{15}=7.24 \times 10^{-10} \mathrm{~m} / \mathrm{V}, s_{11}^{\mathrm{E}}=1.70 \times 10^{-11} \mathrm{~Pa}^{-1}$, $s_{33}^{\mathrm{E}}=2.29 \times 10^{-11} \mathrm{~Pa}^{-1}, s_{12}^{\mathrm{E}}=-5.78 \times 10^{-12} \mathrm{~Pa}^{-1}$, $s_{13}^{\mathrm{E}}=8.79 \times 10^{-12} \mathrm{~Pa}^{-1}, s_{44}^{\mathrm{E}}=3.80 \times 10^{-11} \mathrm{~Pa}^{-1}$,

$$
\begin{aligned}
& s_{66}^{\mathrm{E}}=4.56 \times 10^{-11} \mathrm{~Pa}^{-1}, \frac{\varepsilon_{11}^{S}}{\varepsilon_{0}}=2.44 \times 10^{3}, \\
& \frac{\varepsilon_{33}^{S}}{\varepsilon_{0}}=2.87 \times 10^{3} .
\end{aligned}
$$

First, modal analyses were performed for the first six mode shapes. For further analyses four modes were taken (as the second and third modes as well as the fifth and sixth modes have almost the same frequency due to small influence of piezoelectric elements and symmetry of the plate). Fig. 4 shows the mode shapes chosen for harmonic analyses.

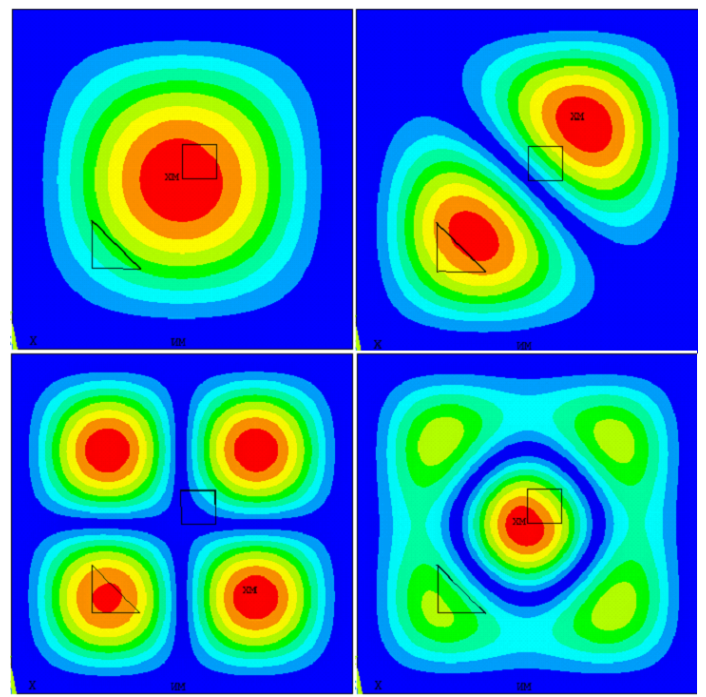

Fig. 4. The modes selected for harmonic analysis.

The amplitude applied to the element used for plate excitation was assumed to be $\mathrm{V}_{e}=100 \mathrm{~V}$. The reduction of plate vibration was obtained using internal ANSYS optimization procedures, with the goal function in the form

$$
|\boldsymbol{J}|=\min \sum_{i=1}^{n} \boldsymbol{X}(i)_{\mathrm{sum}},
$$

where $\boldsymbol{X}(i)_{\text {sum }}$ denotes displacement vector sum in the $i$-th node and $n$ is the number of nodes (in this case all nodes making up the base of the plate).

There were two design variables for the procedure: $V_{a}$ - amplitude of voltage applied to the actuator and $\phi_{a}-$ phase angle of voltage applied to the actuator. The maximum number of steps for the procedure was 30. After each completion the procedure was repeated with design variables ranges being narrowed with the final ranges of $5 \mathrm{~V}$ for $V_{a}$ and $2^{\circ}$ for $\phi_{a}$.

The harmonic analyses were performed for two cases:

1. voltage applied to the whole actuator - analyses were performed for each location of the segment and each material configuration;

2. voltage applied to the "outer" segment of the element and no voltage applied to the "inner" segment of the element (as a electrical separation of the elements) - performed for central placement of the 
"inner" segment in both square and the right-angle triangle based elements, and side placement of the element in right-angle triangle based actuator. It should be noted also that for this case, the material constants used to model the elements were as in PZ29.

\section{Results}

Table I shows the obtained vibration reduction for modeled piezoelements. The first column corresponds to the investigated modes (Fig. 4). The second gives the materials composition of inner and outer segments for two-part actuators or composition of the whole actuator in the case of homogeneous ones. Square and triangle are the shapes of modeled actuators. Headings "Center", "Side", and "Corner" correspond to location of the inner segment in piezoelement. " $0 \mathrm{~V}$ " is a case where the voltage applied to the inner segment of the square based element is always $0 \mathrm{~V}$. " $0 \mathrm{~V} \mathrm{c}$ " and " $0 \mathrm{~V}$ s" represent results for the same situation but with triangle based elements with center and side placement of the inner segment, respectively. For square based homogeneous elements, there is only one result regardless of inner segment placement - that is because the models are identical. For triangle shaped elements, each model had a slightly different mesh, therefore the results could slightly vary (hence even for homogeneous actuators each case has a result written separately).

Looking at the results obtained for the square based piezoactuators it can be seen that there are not any significant differences between the vibration reduction obtained for different placement of inner segment (Table II, Fig. 5). There are two instances that the difference between the best and worst result for the same mode and material composition is greater than $1 \mathrm{~dB}$. But when looked upon other results it is clear that this can be attributed to the optimization algorithm not finding the best possible amplitude and phase of voltage applied to the actuator (with the differences being a fraction of volt and degree, respectively).

When we look at the reduction obtained for the piezoelement with only the outer segment working as an actuator $(0 \mathrm{~V})$ it can be seen that despite smaller working area its effectiveness does not change.

When looking at the results for triangle shaped elements again no significant differences can be found (Table I, Fig. 6). In one instance, the difference between the best and worst result is almost $2 \mathrm{~dB}$, but this is for homogeneous element (where the results should be identical). Again, this can be attributed to the used optimization algorithm and possibly to differences in meshes.

Similarly to square based elements when only the outer segment were used, the obtained results are almost the same regardless of placement of the inner segment.

From this it can be seen that although using a step change of material constants does not improve efficiency of the piezoactuators, it might be possible to have a sensor-actuator by electrically separating some by means of electrical separation of a part of the piezoelement.

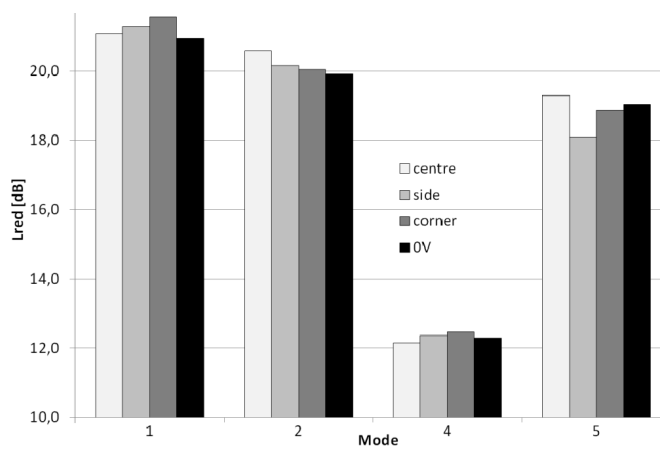

Fig. 5. Results of plate vibration reduction for square based piezoactuators modelled as PZ29 for no voltage applied and PZ29/PZ28 for center, side, and corner location of the inner segment.

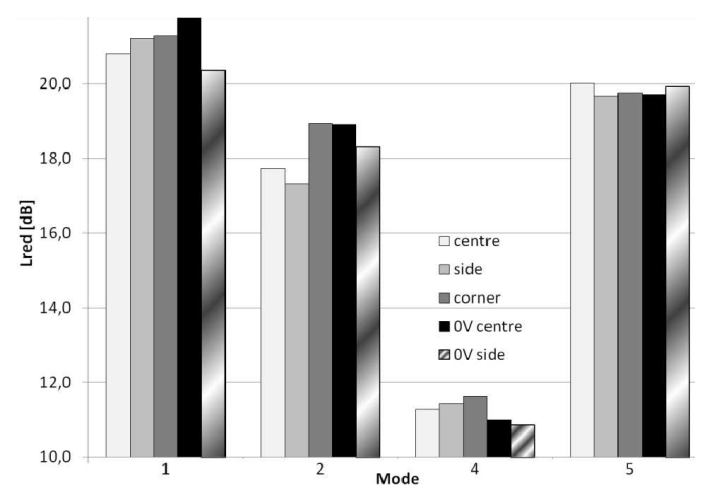

Fig. 6. Results of plate vibration reduction for rightangle triangle based piezoactuators modeled as PZ29 for $0 \mathrm{~V}$ center and 0V side and PZ29/PZ28 for centre, side, and corner location of the inner segment.

Table II shows the results of voltage efficiency of the modeled piezoelements. The columns are the same as in Table I.

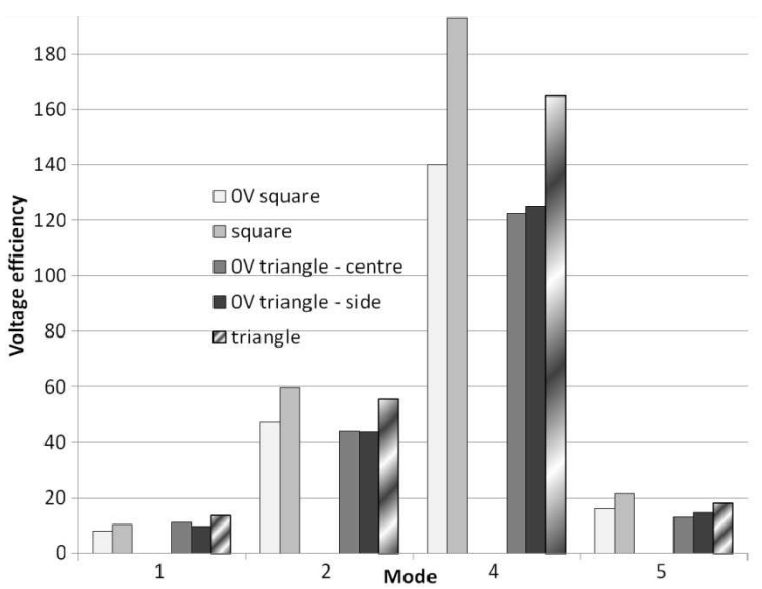

Fig. 7. Voltage efficiency for actuators modeled as PZ29. 
Results of numerical simulation.

TABLE I

\begin{tabular}{|c|c|c|c|c|c|c|c|c|c|c|}
\hline \multirow{2}{*}{ Mode } & \multirow{2}{*}{$\begin{array}{l}\text { Material } \\
\text { (in./out.) }\end{array}$} & \multicolumn{4}{|c|}{ Square } & \multicolumn{5}{|c|}{ Triangle } \\
\hline & & Center & Side & Corner & $0 \mathrm{~V}$ & Center & Side & Corner & $0 \mathrm{~V} \mathrm{c}$ & $0 \mathrm{~V} \mathrm{~s}$ \\
\hline \multirow{4}{*}{1} & PZ29/PZ28 & 21.1 & 21.3 & 21.6 & - & 20.8 & 21.2 & 21.3 & - & - \\
\hline & $\mathrm{PZ28/PZ29}$ & 21.4 & 20.7 & 21.6 & - & 21.1 & 21.0 & 22.0 & - & - \\
\hline & PZ28 & \multicolumn{3}{|c|}{21.2} & - & 21.1 & 22.1 & 22.1 & - & - \\
\hline & PZ29 & \multicolumn{3}{|c|}{20.6} & 21.0 & 20.0 & 20.1 & 20.2 & 21.8 & 20.4 \\
\hline \multirow{4}{*}{2} & PZ29/PZ28 & 20.6 & 20.2 & 20.1 & - & 17.7 & 17.3 & 18.9 & - & - \\
\hline & PZ28/PZ29 & 20.8 & 20.6 & 19.4 & - & 17.4 & 17.3 & 18.9 & - & - \\
\hline & PZ28 & \multicolumn{3}{|c|}{19.4} & - & 18.6 & 16.7 & 18.0 & - & - \\
\hline & PZ29 & \multicolumn{3}{|c|}{18.7} & 19.9 & 17.6 & 16.6 & 17.7 & 18.9 & 18.3 \\
\hline \multirow{4}{*}{4} & PZ29/PZ28 & 12.2 & 12.4 & 12.5 & - & 11.3 & 11.4 & 11.6 & - & - \\
\hline & PZ28/PZ29 & 12.0 & 12.0 & 11.9 & - & 11.5 & 11.2 & 11.1 & - & - \\
\hline & PZ28 & \multicolumn{3}{|c|}{12.5} & - & 11.6 & 11.5 & 11.6 & - & - \\
\hline & PZ29 & \multicolumn{3}{|c|}{12.4} & 12.3 & 10.9 & 10.8 & 10.9 & 11.0 & 10.9 \\
\hline \multirow{4}{*}{5} & PZ29/PZ28 & 19.3 & 18.1 & 18.9 & - & 20.0 & 19.7 & 19.8 & - & - \\
\hline & PZ28/PZ29 & 19.1 & 18.7 & 18.8 & - & 19.6 & 19.9 & 19.8 & - & - \\
\hline & PZ28 & \multicolumn{3}{|c|}{18.9} & - & 19.7 & 19.6 & 19.8 & - & - \\
\hline & PZ29 & \multicolumn{3}{|c|}{18.7} & 19.0 & 19.7 & 19.8 & 19.8 & 19.7 & 19.9 \\
\hline
\end{tabular}

Voltage efficiency.

TABLE II

\begin{tabular}{|c|c|c|c|c|c|c|c|c|c|c|}
\hline \multirow{2}{*}{ Mode } & \multirow{2}{*}{$\begin{array}{l}\text { Material } \\
\text { (in./out.) }\end{array}$} & \multicolumn{4}{|c|}{ Square } & \multicolumn{5}{|c|}{ Triangle } \\
\hline & & Center & Side & Corner & $0 \mathrm{~V}$ & Center & Side & Corner & $0 \mathrm{~V} \mathrm{c}$ & $0 \mathrm{~V} \mathrm{~s}$ \\
\hline \multirow{4}{*}{1} & PZ29/PZ28 & 7.1 & 7.4 & 7.5 & - & 9.7 & 10.2 & 10.0 & - & - \\
\hline & $\mathrm{PZ28/PZ29}$ & 9.4 & 8.9 & 9.2 & - & 12.6 & 12.2 & 13.0 & - & - \\
\hline & PZ28 & \multicolumn{3}{|c|}{5.8} & - & 8.2 & 8.5 & 8.5 & - & - \\
\hline & PZ29 & \multicolumn{3}{|c|}{10.4} & 7.9 & 13.5 & 13.5 & 13.6 & 11.2 & 9.7 \\
\hline \multirow{4}{*}{2} & PZ29/PZ28 & 44.0 & 43.1 & 42.9 & - & 38.5 & 37.7 & 40.1 & - & - \\
\hline & PZ28/PZ29 & 57.4 & 56.8 & 54.0 & - & 47.6 & 48.8 & 53.6 & - & - \\
\hline & PZ28 & \multicolumn{3}{|c|}{33.5} & - & 32.7 & 29.5 & 31.4 & - & - \\
\hline & PZ29 & \multicolumn{3}{|c|}{59.6} & 47.4 & 55.7 & 53.6 & 56.6 & 43.9 & 43.8 \\
\hline \multirow{4}{*}{4} & PZ29/PZ28 & 124.3 & 125.1 & 125.3 & - & 117.6 & 117.1 & 116.0 & - & - \\
\hline & PZ28/PZ29 & 159.4 & 163.0 & 163.2 & - & 150.7 & 151.0 & 151.4 & - & - \\
\hline & PZ28 & \multicolumn{3}{|c|}{103.0} & - & 97.4 & 99.1 & 97.2 & - & - \\
\hline & PZ29 & \multicolumn{3}{|c|}{193.1} & 140.1 & 164.9 & 170.1 & 165.7 & 122.5 & 125.3 \\
\hline \multirow{4}{*}{5} & PZ29/PZ28 & 15.0 & 13.7 & 14.0 & - & 12.8 & 12.1 & 11.6 & - & - \\
\hline & PZ28/PZ29 & 19.1 & 18.9 & 19.4 & - & 15.3 & 16.8 & 16.7 & - & - \\
\hline & PZ28 & \multicolumn{3}{|c|}{11.8} & - & 9.9 & 10.0 & 10.0 & - & - \\
\hline & PZ29 & \multicolumn{3}{|c|}{21.3} & 16.2 & 18.2 & 18.8 & 18.5 & 12.9 & 14.8 \\
\hline
\end{tabular}

The voltage efficiency was a parameter created as a fast and easy although yet simplified way to compare the energy efficiency of different shapes and composition of the modeled piezoelements. It was based on the fact that each element has that same base area $A=1600 \mathrm{~mm}^{2}$. The parameter is defined as

$$
\frac{V}{L}=\frac{L_{\mathrm{red}} V_{e}}{V_{a}}
$$

where $L_{\text {red }}$ denotes obtained vibration reduction, $V_{e}$ is the voltage (amplitude) applied to the piezoelement used for plates excitation, and $V_{a}$ denotes voltage (amplitude) applied to the piezoactuator.

The first thing that can be seen is that the voltage efficiency is proportional to the effective material constants of the piezoactuator. As there were no significant differ- ences between obtained vibration reduction levels, this is what could be expected. The second thing is that except for the first mode the square based elements have slightly (about 10-12\%) higher voltage efficiency. The smaller voltage efficiency for the first mode can be explained by the mode shape and placement of piezoactuators. As the maximum deflection for this mode appears at the center of the plate (Fig. 4) and the actuators were placed so that the centers of their bases are in the same place the edges of the triangle based element are closer to the center of the plate, than the edges of the square based elements.

The results of comparison of voltage efficiency between a "full" actuator and the one in which only the "outer" segment is used are shown in Fig. 7. For this comparison elements modeled as homogeneous PZ29 were chosen. 
Depending on the mode and the shape of actuator the difference between them ranges from $18 \%$ to $29 \%$ with the average being about $24 \%$. This is about the same as the area ratio between the "outer" and "inner" segment. Therefore it can be speculated that reducing the dimensions of the "inner" segment could also proportionally reduce this difference. That way it could be possible to have a sensor-actuator showing only a minimum loss of energy efficiency on the actuator part.

It is worth mentioning that transition of such a complex model to a physical system often requires to construct methods for system response identification [11].

\section{Conclusions}

Numerical analysis of the effects that placement of the "inner" segment in two-part of chosen shapes of piezoactuators and using only a part of the actuator have on their efficiency in plates vibration reduction was presented.

An average reduction of about $18 \mathrm{~dB}$ for square shaped and $17.5 \mathrm{~dB}$ for right-angle triangle shaped actuators was obtained.

The performed simulations showed that changing the location of the "inner" segment of a two-part piezoelement do not improve its effectiveness and with the results of previous simulations it can be said that two-part piezoactuators are not an improvement over homogeneous one (although this should be verified experimentally).

It has been also shown that using only a part of piezoelement (in this case, the "outer" segment) also does not change the obtained reduction. The voltage needed to obtained these results needs to be higher (for modeled elements, an average of $24 \%$ increase in voltage was required) but it can be assumed that reducing the inert part of the actuator should decrease the needed voltage.
Therefore it can be assumed that by separating a part of the piezoactuator electrically it is possible to obtain a sensor-actuator without decreasing its effectiveness in its vibration reduction and with minimal increase in voltage applied to it (although this needs further testing).

\section{Acknowledgments}

This study is a part of the research project N N504078038 supported by Polish Ministry of Science and Higher Education.

\section{References}

[1] E.K. Dimitriadis, C.R. Fuller, C.A. Rogers, J. Vib. Acoust. 113, 100 (1991).

[2] A. Branski, Acta Phys. Pol. A 123, 1123 (2013).

[3] U. Ferdek, M.S. Kozien, Acta Phys. Pol. A 123, 1044 (2011).

[4] M.S. Kozień, Acta Phys. Pol. A 123, 1029 (2013).

[5] D. Iwanski, J. Wiciak, Acta Phys. Pol. A 119, 972 (2011).

[6] R. Trojanowski, J. Wiciak, Acta Phys. Pol. A $\mathbf{1 1 8 ,}$ 168 (2010).

[7] A. Branski, S. Szela, Acta Phys. Pol. A 119, 942 (2011).

[8] M. Wiciak, Acta Phys. Pol. A 121, A-142 (2012).

[9] C.T. Loy, K.Y. Lam, J.N. Reddy, Int. J. Mech. Sci. 41, 309 (1999).

[10] M. Pietrzakowski, Mechanics 26(4), 181 (2007).

[11] A. Ozga, Acta Phys. Pol. 123, 1034 (2013). 